\title{
An Operational Characterization of Strong Normalization ${ }^{\star}$
}

\author{
Luca Paolini $^{1}$, Elaine Pimentel ${ }^{2}$, and Simona Ronchi Della Rocca ${ }^{1}$ \\ 1 Dipartimento di Informatica, Università di Torino (Italy) \\ 2 Departamento de Matemática, Universidade Federal de Minas Gerais (Brazil)
}

\begin{abstract}
This paper introduces the $\Phi$-calculus, a new call-by-value version of the $\lambda$-calculus, following the spirit of Plotkin's $\lambda \beta_{v}$-calculus. The $\Phi$-calculus satisfies some interesting properties, in particular that its set of solvable terms coincides with the set of $\beta$-strongly normalizing terms in the classical $\lambda$-calculus.
\end{abstract}

\section{Introduction}

The standard $\lambda$-calculus equipped with the $\beta$-reduction is the paradigmatic language for the call-by-name functional computation. Its call-by-value version, historically called $\lambda \beta_{v}$-calculus, has been introduced by Plotkin in 1975 11. The $\lambda \beta_{v}$-calculus is based on a restriction of the $\beta$-rule, firing it only when the argument belongs to a particular subset of terms, called values. In [10,13, two co-authors of this paper, in order to treat these two different calculi in a uniform way, introduced the $\lambda \Delta$-calculus, parametric with respect to a subset $\Delta$ of terms, called input values, which generalizes the idea of Plotkin's values. Some conditions on $\Delta$ have been stated assuring some good properties for the calculus, in particular confluence and standardization. These conditions are very natural: the set of input values must contain the set of variables and it must be closed under substitution and $\Delta$-reduction (a further condition is necessary for having standardization). Note that, in this setting, the standard $\lambda$-calculus now can be seen as a degenerated case of a $\lambda \Delta$-calculus, where all terms are input values (that is, the set of input values is $\Lambda$ ). Plotkin's calculus coincides with the $\lambda \Gamma$-calculus, where $\Gamma=\operatorname{Var} \cup\{\lambda x . M \mid M \in \Lambda\}$.

The formalization of the $\lambda \Delta$-calculus, where both call-by-name and call-byvalue calculus can be uniformly representable, is an useful tool for studying the relationship between these two notions of computations. Some interesting properties relating $\lambda \Lambda$ and $\lambda \Gamma$-calculus have been already proved. For example, it turns out that, in the $\lambda \Gamma$-calculus, the notion of normal form is meaningless since there are different $\Gamma$-normal forms that can be consistently equated (see [13]). But the notion of $\beta$-normal form has an important meaning also in this calculus: in [7] it was proved that two different $\beta \eta$-normal forms can be separated in the $\lambda \Gamma$-calculus, and hence they cannot be consistently equated in any model.

In 8 we further explored the relationship between $\beta$-normal forms and $\Gamma$ evaluation. In that paper, it was proved that the set of strongly $\beta$-normalizing

\footnotetext{
` Paper partially supported by MIUR-PRIN'04 FOLLIA Project and by CNPq.
} 
terms, with respect to a lazy reduction 1 , coincides with the set of potentially $\Gamma$-valuable terms. A term is potentially $\Delta$-valuable (where $\Delta$ is any set of input values) if and only if there is a substitution, replacing variables by closed input values, such that the substituted term reduces to an input value. Being input values the only terms that can be manipulated by the $\Delta$-calculus (and that can be argument of a function) this class of terms is particularly interesting. For example, in Plotkin's operational semantics for the $\lambda \Gamma$-calculus [1] based on the SECD machine [6], a potentially $\Gamma$-valuable term is always different from a non potentially $\Gamma$-valuable term. Moreover, all the non potentially $\Gamma$-valuable terms are equated 13 .

A first natural question that arises is if this analogy between a call-by-name strong normalization and a call-by-value evaluation can be further developed. In particular, we are interested to know if there is a set of input values $\Phi$ such that the set of potentially $\Phi$-valuable terms coincides with the set of strongly $\beta$-normalizing terms.

The set of lazy strong $\beta$-normalizing terms and the set $\Gamma$ of input values have an interesting structural analogy: in order to find a lazy $\beta$-normal form of a $\lambda$-term it is not necessary to reduce under a $\lambda$-abstraction. The same happens when checking if a $\lambda$-term is a $\Gamma$-input value: it is not necessary to look under a $\lambda$-abstraction since all $\lambda$-abstractions are in $\Gamma$. We will call weak any set of input values containing all $\lambda$-abstractions. It turns out that "to be weak" has some consequences. Extending the notion of solvability to a generic $\Delta$-calculus in the natural way, a term $M$ is called $\Delta$-solvable if and only if there is a sequence $P_{1}, \ldots, P_{n}$ of $\Delta$-input values such that $(\lambda \vec{x} . M) P_{1} \ldots P_{n}={ }_{\Delta} \lambda x . x$ (where $\lambda \vec{x} . M$ is the term obtained from $M$ by abstracting it with respect to all its free variables). In the $\lambda \Gamma$-calculus, the set of solvable terms is a proper subset of the set of potentially valuable terms. This is a consequence of the fact that $\Gamma$ is weak: if $U$ is a closed $\Gamma$-unsolvable term then $\lambda x . U \in \Gamma$ is a potentially valuable, but unsolvable term.

This yields to a second question: is there a $\lambda \Delta$-calculus such that the set of $\Delta$-potentially valuable terms coincides with the set of $\Delta$-solvable terms?

Certainly, such a $\Delta$ could not be weak. In fact, in order to check if a term is solvable, it is necessary to perform the evaluation under the $\lambda$-abstraction.

In this paper, we give an answer to the two questions posted above, presenting the $\lambda \Phi$-calculus, where $\Phi$ is a set of input values which is the minimal solution of a recursive equation. $\Phi$ is not weak, since it is a proper subset of the set of $\Phi$-normal forms. It turns out that the $\lambda \Phi$-calculus enjoys, besides confluence, the standardization property. Moreover, $\Phi$ is a proper subset of the set of strongly $\beta$-normalizing terms, and we prove that $\Phi$ is minimal between all sets of terms answering the first question. Hence our result can be rephrased as: the whole set

${ }^{1}$ Following [13], lazy $\beta$-reduction is defined as the closure of the $\beta$-rule under application, but not under abstraction. This corresponds operationally to do not perform reduction under the $\lambda$-abstraction. In the field of real functional languages, "lazy" is used with a different meaning. 
of strongly $\beta$-normalizing terms can be operationally described through a proper subset of it.

A further comment is in order. Plotkin's motivation on designing the $\lambda \Gamma$ calculus was to propose a paradigmatic language for the call-by-value evaluation in real programming languages and, from this point of view, the choice of a weak set of input values is natural for modeling the notion of closure in the sense of Landin [6]. On the other hand, our motivation is purely theoretical, and the $\lambda \Phi$-calculus presented here is not an alternative proposal for designing new call-by-value languages. In any case, implementing the $\Phi$-calculus would be difficult, being the set $\Phi$ just semi-decidable. But we believe that this study is interesting by itself. In fact, Plotkin in 11, posed the question of an existence of call-by-value $\lambda$-language alternative to $\lambda \Gamma$. He said that the natural proposal was to choose the set of the $\beta$-normal forms as an alternative to $\Gamma$. Unfortunately, the set of $\beta$-normal forms induces a calculus lacking the confluence property, in fact $\beta$-normal forms are not input values in our sense. Hence the $\lambda \Phi$-calculus, enjoying both confluence and standardization, gives an answer to this further question as well.

The rest of the paper is organized as follows: Section 2 contains basic notions of the parametric $\lambda \Delta$-calculus; in Section 3 the $\lambda \Phi$-calculus is introduced and finally, in Section 4 the main theorem is stated and proved.

\section{The Parametric $\lambda$-Calculus}

A calculus is a language equipped with some reduction rules. We will consider here calculi sharing the same language, the language of $\lambda$-calculus, while they differ from each other in their reduction rules. In order to treat them in a uniform way we will use the notion of parametric calculus, the $\lambda \Delta$-calculus, that gives rise to different calculi by different instantiations of the parameter $\Delta$. The $\lambda \Delta$ calculus has been studied in [10, 13]. We use the terminology of [2,13].

Definition 1 (The language $\Lambda$ ). Let Var be a countable set of variables. The set $\Lambda$ of $\lambda$-terms is defined by the following grammar:

$$
M::=x|M M| \lambda x \cdot M
$$

$\lambda$-terms will be ranged over by Latin capital letters. Sets of $\lambda$-terms will be denoted by Greek capital letters. If $\Theta$ denotes a set of terms $(\Theta)^{0}$ is the set of closed terms belonging to $\Theta$.

Sometimes, we will refer to $\lambda$-terms simply as terms. As usual, terms will be considered modulo $\alpha$-conversion, i.e., modulo names of bound variables. The symbol $\equiv$ will denote syntactical identity of terms, up to $\alpha$-equivalence.

We will use the following abbreviations, in order to avoid an excessive number of parentheses, thereby $\lambda x_{1} \ldots x_{n} . M$ will stand for $\left(\lambda x_{1}\left(\ldots\left(\lambda x_{n} . M\right) \ldots\right)\right)$ and $M N_{1} N_{2} \ldots N_{n}$ will stand for $\left(\ldots\left(\left(M N_{1}\right) N_{2}\right) \ldots N_{n}\right)$. Moreover $\vec{M}$ will denote a sequence of terms $M_{1}, \ldots, M_{n}$, for some $n \geq 0$, and $\lambda \vec{x} . M$ and $\vec{M} \vec{N}$, will denote 
respectively $\lambda x_{1} \ldots x_{n} . M$ and $M_{1} \ldots M_{m} N_{1} \ldots N_{n}$, for some $n, m \geq 0$. The length of the sequence $\vec{N}$ is denoted by $\|\vec{N}\|$.

The $\lambda \Delta$-calculus consists of the language $\Lambda$ equipped with a set $\Delta \subseteq \Lambda$ of input values, satisfying some closure conditions. Informally, input values represent already evaluated terms, that can be passed as arguments. The set $\Delta$ of input values and the reduction $\rightarrow \Delta$, induced by it, are defined below.

Definition 2. Let $\Delta \subseteq \Lambda$.

(i) The $\Delta$-reduction $(\rightarrow \Delta)$ is the contextual closure of the following rule:

$$
(\lambda x . M) N \rightarrow M[N / x] \text { if and only if } N \in \Delta .
$$

$(\lambda x . M) N$ is a $\Delta$-redex (or simply redex).

(ii) $\rightarrow_{\Delta}^{+}, \rightarrow_{\Delta}^{*}$ and $={ }_{\Delta}$ are respectively the transitive closure of $\rightarrow \Delta$, the reflexive and transitive closure of $\rightarrow \Delta$ and the symmetric, reflexive and transitive closure of $\rightarrow \Delta$.

(iii) $A$ set $\Delta \subseteq \Lambda$ is a set of input values, when the following conditions are satisfied:

- $\operatorname{Var} \subseteq \Delta$

- $P, Q \in \Delta$ implies $P[Q / x] \in \Delta$, for each $x \in \operatorname{Var}$

- $M \in \Delta$ and $M \rightarrow \Delta N$ imply $N \in \Delta$

(Var-closure); (substitution closure); (reduction closure).

The closure conditions on the set of input values assure us that the $\lambda \Delta$-calculus enjoys the confluence property for every $\Delta$, i.e., the following theorem holds.

Theorem 3 (Confluence). [10, 13] Let $M \rightarrow{ }_{\Delta}^{*} N_{1}$ and $M \rightarrow{ }_{\Delta}^{*} N_{2}$. There is $Q$ such that both $N_{1} \rightarrow{ }_{\Delta}^{*} Q$ and $N_{2} \rightarrow_{\Delta}^{*} Q$.

Two particular instantiations of $\Delta$ give rise to the call-by-name and the callby-value $\lambda$-calculus. The call-by-name $\lambda$-calculus (i.e., the standard $\lambda$-calculus equipped with the $\beta$-reduction) coincides with the $\lambda \Lambda$-calculus. The call-by-value $\lambda$-calculus (defined by Plotkin in 11) coincides with the $\lambda \Gamma$-calculus, where $\Gamma=\operatorname{Var} \cup\{\lambda x \cdot M \mid M \in \Lambda\}$.

Let $\Delta$ be a set of input values. A term of the $\lambda \Delta$-calculus is in $\Delta$-normal form if and only if it does no contain occurrences of $\Delta$-redexes. A term $M$ is strongly $\Delta$-normalizing if both $M$ has a $\Delta$-normal form and every reduction sequence starting from $M$ eventually stops.

The set $\Delta$-NF of $\Delta$-normal forms can be defined in the following recursive way:

$$
\begin{aligned}
\Delta-\mathrm{NF}=\operatorname{Var} & \cup\left\{x M_{1} \ldots M_{n} \mid M_{k} \in \Delta-\mathrm{NF}(1 \leq k \leq n)\right\} \\
& \cup\{\lambda \vec{x} . M \mid M \in \Delta-\mathrm{NF}\} \\
& \cup\left\{(\lambda x . P) Q M_{1} \ldots M_{n} \mid P, Q, M_{k} \in \Delta-\mathrm{NF}, Q \notin \Delta \quad(1 \leq k \leq n)\right\} .
\end{aligned}
$$

Note that for the $\lambda \Lambda$-calculus, being $\Lambda$ its set of input values, the last case cannot happen, i.e., there are no normal forms of the shape $(\lambda x . P) Q M_{1} \ldots M_{n}$, so $\Lambda$-NF $\subseteq \Delta$-NF, for all $\Delta$. 
In the $\lambda \Gamma$-calculus, the notion of normal form is meaningless. In fact, there are different $\Gamma$-normal forms that can be consistently equated. The key notion, in a call by value setting, is the one of (potential) valuability, given in the next definition (see [9], [13]).

Definition 4. (i) A term $M$ is $\Delta$-valuable if and only if there is $N \in \Delta$ such that $M \rightarrow_{\Delta}^{*} N$.

(ii) A term $M$ is potentially $\Delta$-valuable if and only if there is a substitution $\mathbf{s}$, replacing variables by closed terms belonging to $\Delta$, such that $\mathbf{s}(M)$ is $\Delta$ valuable.

It is immediate to verify that a closed term is potentially $\Delta$-valuable if and only if it is $\Delta$-valuable. Note that the notion of $\Delta$-normal form and that one of potentially $\Delta$-valuable are orthogonal. As an example, consider the $\lambda \Gamma$-calculus, and the term $M \equiv(\lambda z . D)(y I) D$, where $I \equiv \lambda x . x$ and $D \equiv(\lambda z . z z) . M$ is in $\Gamma$-normal form, but it is neither an input value nor potentially $\Gamma$-valuable. In fact, consider $M[Q / y]$, for some $Q \in(\Gamma)^{0}$. If $Q I$ reduces to an element in $\Gamma$ then $M[Q / y] \equiv(\lambda z . D)(Q I) D$ reduces to $D D$, which is not an input value. Otherwise $M[Q / y] \rightarrow_{\Gamma}^{*}(\lambda z . D) Q^{\prime} D$, for every $Q^{\prime}$ such that $Q I \rightarrow_{\Gamma}^{*} Q^{\prime}$, which is not an input value. Thus $(\lambda z . D)(Q I) D$ is not $\Gamma$-valuable. We call $\Delta$-liarnormal forms terms which are in $\Delta$-normal form but that are not potentially $\Delta$-valuable.

In the $\lambda \Lambda$-calculus, the notion of solvability plays an important role, since in some sense the solvable terms represent the meaningful computations 2. In 9], $\Gamma$-solvable and potentially $\Gamma$-valuable terms has been characterized. This notion has been extended to the parametric $\lambda \Delta$-calculus in 13 .

Definition 5. (i) A context $C[$.$] is \Delta$-valuable if and only if $C[.] \equiv(\lambda \vec{x} .[].) \vec{P}$ where each $P \in \vec{P}$ is such that $P \in \Delta$.

(ii) A term $M$ is $\Delta$-solvable if and only if there is a $\Delta$-valuable context $C$ [.] such that:

$$
C[M]={ }_{\Delta} I .
$$

(iii) A term is $\Delta$-unsolvable if and only if it is not $\Delta$-solvable.

Note that $(\lambda \vec{x} \cdot M) \vec{N}={ }_{\Delta} I$ means $(\lambda \vec{x} \cdot M) \vec{N} \rightarrow_{\Delta}^{*} I$, since $I$ is in $\Delta$-NF, for every $\Delta$.

\section{The $\lambda \Phi$-Calculus}

As observed in the introduction, we are interested to know if there is a set of input values $\Phi$ such that the set of potentially $\Phi$-valuable terms coincides with the set of strongly $\beta$-normalizing terms. Such a set cannot be weak i.e., it cannot contain all $\lambda$-abstractions. Since input values represent already evaluated terms, the natural choice would be to take $\Delta$ such that $\Delta$ coincides with its set of normal forms (i.e. $\Delta=\Delta-N F$ ). From the recursive definition of $\Delta$-normal form 
restricted to the case where $\Delta$ is a set of input values, the following equations would be obtained:

(1.1) $\Delta=\operatorname{Var} \cup\left\{x M_{1} \ldots M_{n} \mid M_{k} \in \Delta(1 \leq k \leq n)\right\} \cup\{\lambda \vec{x} . M \mid M \in \Delta\} \cup$ $\left\{(\lambda x . P) Q M_{1} \ldots M_{n} \mid P, Q, M_{k} \in \Delta(1 \leq k \leq n), Q \notin \Delta\right\}$

(1.2) $M, P \in \Delta$ implies $M[P / x] \in \Delta$.

Note that the reduction closure for $\Delta$ is trivially satisfied, since we asked that terms in $\Delta$ are $\Delta$-normal forms.

The only solution to the equation (1.1) is $\Delta=\Lambda-N F$. In fact, the set $\left\{(\lambda x . P) Q M_{1} \ldots M_{n} \mid P, Q, M_{k} \in \Delta(1 \leq k \leq n), Q \notin \Delta\right\}$ is empty, due to the contradictory condition on $Q$. Unfortunately, $\Delta=\Lambda-N F$ does not satisfy the equation (1.2).

On the other hand, the simpler way of forcing $\Delta$ to satisfy equation (1.2) would be to restrict $\Delta$ so that it contains, besides variables, only closed terms. That is, to choose $\Delta^{\dagger}=(\Lambda-N F)^{0} \cup \operatorname{Var}$. But $\Delta^{\dagger}$ does not solve our problem. In fact, the term $I(\lambda x . I(x x))$ is a strongly $\Lambda$-normalizing term, but it is not $\Delta^{\dagger}$-solvable. Indeed $I(\lambda x . I(x x))$ is a $\Delta^{\dagger}$-NF which cannot be reduced (in the $\lambda \Delta^{\dagger}$-calculus $)$, since $\lambda x . I(x x) \notin \Delta^{\dagger}$.

The discussion above suggests that we should look for a set $\Delta$ which is a proper subset of the $\Delta$-normal forms, and a proper superset of $(\Lambda-N F)^{0} \cup \operatorname{Var}$. Let us maintain the choice that $\Delta$ contains, besides variables, only closed terms. The previous pair of equations now become:

$$
\begin{aligned}
& \Theta=\operatorname{Var} \cup\left\{x M_{1} \ldots M_{n} \mid M_{k} \in \Theta(1 \leq k \leq n)\right\} \cup\{\lambda \vec{x} . M \mid M \in \Theta\} \cup \\
& \left\{(\lambda x . P) Q M_{1} \ldots M_{n} \mid P, Q, M_{1} \ldots M_{n} \in \Theta Q \notin \Delta\right\} \\
& \Delta=\operatorname{Var} \cup(\Theta)^{0} .
\end{aligned}
$$

Note that $\Theta=\Delta$-NF and $\Delta$ is a set of input values.

But the last condition on equation (2.1) is too weak again, since now the set $\Theta$ may contain some $\Delta$-liar-normal forms. As an example, $M \equiv(\lambda z . D)(y I) D$, where $D \equiv(\lambda z . z z)$, is a $\Delta$-liar-normal form satisfying both the previous equations. Since $\Delta$-liar-normal forms are $\Delta$-unsolvable, such a set cannot supply an answer to our second question, i.e., cannot be such that the set of $\Delta$-solvable terms coincide with the set of $\Delta$-potentially valuable terms.

The following easy to prove property will help us on excluding such dangerous terms.

Property 6. If there is a substitution s such that $\mathbf{s}(P[Q / x] \vec{M}) \rightarrow_{\Delta}^{*} R \in \Delta$ and $\mathbf{s}(Q) \rightarrow{ }_{\Delta}^{*} Q^{\prime} \in \Delta$ then $\mathbf{s}((\lambda x . P) Q \vec{M}) \rightarrow{ }_{\Delta}^{*} R$.

Taking into account the previous property, the pair of equations now becomes:

$$
\begin{aligned}
(3.1) & \Theta=\operatorname{Var} \cup\left\{x M_{1} \ldots M_{n} \mid M_{k} \in \Theta(1 \leq k \leq n)\right\} \cup\{\lambda \vec{x} . M \mid M \in \Theta\} \cup \\
& \left\{(\lambda x . P) Q M_{1} . . M_{n} \mid Q, M_{1}, \ldots, M_{n} \in \Theta, Q \notin \Delta, P[Q / x] M_{1} \ldots M_{n} \rightarrow^{*}{ }_{\Delta} R \in\right. \\
& \Theta\} ; \\
\text { (3.2) } & \Delta=\operatorname{Var} \cup(\Theta)^{0} .
\end{aligned}
$$

The minimal solution of this pair of recursive equations is defined next. 
Definition 7. The sets of $\lambda$-terms $\Upsilon_{i}, \Phi_{i}(i \in \mathbb{N})$ are defined by mutual induction, as follows

$$
\begin{aligned}
& \Upsilon_{0}=\operatorname{Var} \\
& \Phi_{i} \quad=\operatorname{Var} \cup\left(\Upsilon_{i}\right)^{0} \\
& \Upsilon_{i+1}=\operatorname{Var} \cup\left\{x M_{1} \ldots M_{n} \mid M_{k} \in \Upsilon_{i}(1 \leq k \leq n)\right\} \cup\left\{\lambda \vec{x} . M \mid M \in \Upsilon_{i}\right\} \\
& \cup\left\{\begin{array}{l|l}
(\lambda x . P) Q M_{1} \ldots M_{n} & \begin{array}{l}
Q \in \Upsilon_{i}-\left(\Lambda^{0} \cup \operatorname{Var}\right), \quad M_{1}, \ldots, M_{n} \in \Upsilon_{i}, \\
P[Q / x] M_{1} \ldots M_{n} \rightarrow_{\Phi_{i}}^{*} R \in \Upsilon_{i}
\end{array}
\end{array}\right\}
\end{aligned}
$$

Moreover, we define $\Upsilon=\cup_{i} \Upsilon_{i}$ and $\Phi=\operatorname{Var} \cup(\Upsilon)^{0}$.

For example, $\Phi_{0}=\operatorname{Var}, \Upsilon_{1}=\operatorname{Var} \cup\left\{x y_{1} \ldots y_{n} \mid y_{i} \in \operatorname{Var}\right\} \cup\{\lambda \vec{x} . y \mid y \in \operatorname{Var}\}$ and $\Phi_{1}=\operatorname{Var} \cup\left\{\lambda x_{1} \ldots x_{m} . x_{j} \mid 1 \leq j \leq m\right\}$.

The following result holds trivially:

Lemma 8. (i) For all $i \in \mathbb{N}, \Phi_{i}$ is a set of input value.

(ii) $\Phi=\cup_{i} \Phi_{i}$.

(iii) $\Phi$ is a set of input values.

(iv) For all $i \in \mathbb{N}, \Upsilon$ and $\Upsilon_{i}$ are not sets of input values.

Also, it is easy to check that the following properties hold.

Property 9. (i) $\Upsilon_{i} \subset \Upsilon_{i+1}, \Phi_{i} \subset \Phi_{i+1}$ and $\rightarrow_{\Phi_{i}} \subseteq \rightarrow_{\Phi_{i+1}}$, for all $i \in \mathbb{N}$;

(ii) $\Upsilon_{i} \subset \Upsilon, \Phi_{i} \subset \Phi$ and $\rightarrow_{\Phi_{i}} \subseteq \rightarrow_{\Phi}$, for all $i \in \mathbb{N}$;

(iii) $\Upsilon^{0}=\Phi^{0}$;

(iv) $M \in \Phi^{0}$ implies $M \equiv \lambda z . P$, for some $z \in \operatorname{Var}$ and $P \in \Upsilon$ (note that $\Phi \subseteq \Gamma$ );

(v) $\Phi \subseteq \Upsilon$ and $\Upsilon \subseteq \Phi$-NF;

(vi) $\Phi$-NF $\nsubseteq \Upsilon$ and $\Phi$-NF $\nsubseteq \Phi$.

Proof. (vi) Let $M \equiv \lambda z .(\lambda x . D)(z I) D . M \in \Phi$-NF since $z I \notin \Phi$. But $M \notin \Upsilon$ and $M \notin \Phi$.

Lemma 8 (iii) implies that the $\lambda \Phi$-calculus enjoys the confluence property. Moreover it is possible to check that it also satisfies the additional necessary condition for standardization, stated in [10,13].

\section{The Main Result}

The $\lambda \Phi$-calculus, besides confluence and standardization, has some further interesting properties. The most important one is that the set of potentially $\Phi$ valuable terms coincides with the set of strongly $\Lambda$-normalizing terms. Other properties characterize completely the operational behavior of the $\lambda \Phi$-calculus. In particular, a term is $\Phi$-solvable if and only if it is potentially $\Phi$-valuable if and only if it $\Phi$-reduces to a term in $\Upsilon$.

Theorem 10 (Main Theorem). The following statements are equivalent:

(i) $M$ is strongly $\Lambda$-normalizing;

(ii) $M$ is $\Phi$-solvable;

(iii) $M$ is potentially $\Phi$-valuable;

(iv) $M \rightarrow_{\Phi}^{*} R \in \Upsilon$; 
Proof. (i) implies (ii) by Theorem 26.

(ii) implies (iii) by Theorem [16] (ii).

(iii) implies (iv) by Theorem [16.(i).

(iv) implies (i) by Theorem 20.

where theorems 16, 20 and 26] will be proved later in this Section.

Notice that $\Phi$ is a proper subset of the set of strongly $\Lambda$-normalizing terms. In fact, a strongly $\Lambda$-normalizing term of the shape $M_{1} \ldots M_{2}$ where $M_{i}$ is closed $(1 \leq i \leq n)$ does not belong to $\Phi$. Moreover $\Phi$ is minimal between all sets of input values which answer our first question.

Property 11. Let $\Delta^{\star}$ be a set of input values.

If the set of potentially $\Delta^{\star}$-valuable terms coincides with the set of the strongly $\Lambda$-normalizing terms and $\Delta^{\star} \subset \Phi$ then $\Delta^{\star}=\Phi$.

Proof. Clearly $\Delta^{\star}=\Phi$ if and only if $\left(\Delta^{\star}\right)^{0}=\Phi^{0}$, since $\Delta^{\star} \subset \Phi$ and $\Phi=\operatorname{Var} \cup \Phi^{0}$. Thus suppose $M \in \Phi^{0}$. Note that $M$ is $\Phi$-valuable, potentially $\Phi$-valuable and also in $\Phi$-normal form. Moreover $M$ is a closed strongly $\Lambda$-normalizing term, by the Main Theorem. Thus, $M$ is potentially $\Delta^{\star}$-valuable by hypothesis and $M \in \Lambda^{0}$ implies that $M$ is $\Delta^{\star}$-valuable. But $\Delta^{\star} \subset \Phi$ implies $\Phi$-NF $\subset \Delta^{\star}$-NF, hence $M \in \Delta^{\star}$-NF. Then $M \in \Delta^{\star}$ and the proof is done.

Finally, it is worthy to say that, although $\Phi$ is minimal, it is not the minimum set supplying an answer to questions stated in the introduction. In fact, the minimum solution to the following equations:

$$
\begin{aligned}
\Theta= & \left\{\lambda x_{0} \ldots x_{n} . y \mid y \neq x_{i} \quad(0 \leq i \leq n)\right\} \cup \\
& \left\{x M_{1} \ldots M_{n} \mid M_{k} \in \Theta(1 \leq k \leq n)\right\} \cup\{\lambda \vec{x} \cdot M \mid M \in \Theta\} \cup \\
& \left\{(\lambda x . P) Q M_{1} \ldots M_{n} \mid Q, M_{1}, \ldots, M_{n} \in \Theta, Q \notin \Delta, P[Q / x] M_{1} \ldots M_{n} \rightarrow^{*}{ }_{\Delta} R \in \Theta\right\} \\
\Delta= & \left\{\lambda x_{0} \ldots x_{n} . y \mid y \neq x_{i} \quad(0 \leq i \leq n)\right\} \cup(\Theta)^{0}
\end{aligned}
$$

also answers our questions and it is not comparable with $\Phi$.

The rest of the paper is devoted to the proof of the Main Theorem.

\subsection{Potential $\Phi$-Valuability and $\Phi$-Solvability}

First of all, we will introduce the weight of a terms, as measure for carrying out some inductive proofs. The weight of a term $M$ is an upper bound to the number of symbols of $M$, to the length of its leftmost $\Lambda$-reduction sequence and to the length of its $\Phi$-reduction sequence according to the standard strategy [10].

Definition 12. The weight $\left\langle-_{-}\right\rangle: \Lambda \longrightarrow \mathbb{N}$ is the partial function defined as follows:

$-\left\langle\lambda x \cdot M^{\prime}\right\rangle=1+\left\langle M^{\prime}\right\rangle$.

$-\left\langle x M_{1} \ldots M_{m}\right\rangle=1+\left\langle M_{1}\right\rangle+\ldots .+\left\langle M_{m}\right\rangle$.

$-\left\langle\left(\lambda x . M_{0}\right) M_{1} \ldots M_{m}\right\rangle=1+\left\langle M_{1}\right\rangle+\left\langle M_{0}\left[M_{1} / x\right] M_{2} \ldots M_{m}\right\rangle$.

As examples $\langle x\rangle=1,\langle x x\rangle=2,\langle\lambda x . x x\rangle=3$. It is easy to check that $M \rightarrow_{\Phi} N$ implies $M[P / z] \rightarrow_{\Phi} N[P / z]$, if $P \in \Phi$. 
Lemma 13. (i) If $M \in \Upsilon$ then $\langle M\rangle \in \mathbb{N}$.

(ii) If $M \rightarrow_{\Phi}^{+} N$ and $\langle N\rangle \in \mathbb{N}$ then, both $\langle M\rangle \in \mathbb{N}$ and $\langle N\rangle\langle\langle M\rangle$.

(iii) If $M \rightarrow_{\Phi}^{+} N$ and $\langle M\rangle \in \mathbb{N}$ then, both $\langle N\rangle \in \mathbb{N}$ and $\langle N\rangle\langle\langle M\rangle$.

Proof. (i) The proof can be done by induction on the $\Upsilon$ stratification.

(ii) The proof is given by induction on $\langle N\rangle$.

- If $M \equiv \lambda x . P \rightarrow_{\Phi}^{+} \lambda x . P^{\prime} \equiv N$ then $\left\langle P^{\prime}\right\rangle \in \mathbb{N}$ implies $\left\langle P^{\prime}\right\rangle\langle\langle P\rangle \in \mathbb{N}$ by induction, hence $\langle N\rangle\langle 1+\langle P\rangle=\langle M\rangle$.

- Let $M \equiv x M_{1} \ldots M_{m} \rightarrow_{\Phi}^{+} x N_{1} \ldots N_{m} \equiv N(m \geq 1)$ where either $M_{k} \rightarrow_{\Phi}^{+}$ $N_{k}$ or $M_{k} \equiv N_{k}(1 \leq k \leq m)$. Note that there is at least one $h \in \mathbb{N}$ such that $M_{h} \rightarrow{ }_{\Phi}^{+} N_{h}$ and $\left\langle N_{h}\right\rangle<\left\langle M_{h}\right\rangle(1 \leq h, k \leq m)$. Thus the proof follows easily by induction.

- Let $M \equiv\left(\lambda z \cdot M_{0}\right) M_{1} \ldots M_{m} \rightarrow_{\Phi}^{+} N$ for some $m \geq 1$.

Either $M \rightarrow_{\Phi}^{*}\left(\lambda x . N_{0}\right) N_{1} \ldots N_{m} \rightarrow \Phi N_{0}\left[N_{1} / x\right] N_{1} \ldots N_{m} \rightarrow_{\Phi}^{*} N$ or $M \rightarrow_{\Phi}^{+}$ $\left(\lambda x . N_{0}\right) N_{1} \ldots N_{m} \equiv N$, where $M_{k} \rightarrow \underset{\Phi}{+} N_{k}$ or $M_{k} \equiv N_{k}(1 \leq k \leq m)$. In all cases, the proof follows by induction.

(iii) The proof is given by induction on $\langle M\rangle$.

- If $M \equiv \lambda x . M_{0} \rightarrow_{\Phi}^{+} \lambda x . N_{0} \equiv N$ then $\left\langle M_{0}\right\rangle \in \mathbb{N}$. Hence $\left\langle N_{0}\right\rangle\left\langle\left\langle M_{0}\right\rangle\right.$ by induction, thus $\langle N\rangle=1+\left\langle N_{0}\right\rangle<1+\left\langle M_{0}\right\rangle=\langle M\rangle$.

- Let $M \equiv x M_{1} \ldots M_{m} \rightarrow_{\Phi}^{+} x N_{1} \ldots N_{m} \equiv N(m \geq 1)$, where either $M_{k} \rightarrow_{\Phi}^{+}$ $N_{k}$ or $M_{k} \equiv N_{k}(1 \leq k \leq m)$. Note that there is $h \in \mathbb{N}$ such that $M_{h} \rightarrow_{\Phi}^{+} N_{h}$ and $\left\langle N_{h}\right\rangle<\left\langle M_{h}\right\rangle$. Thus the proof follows easily by induction.

- Let $M \equiv\left(\lambda z \cdot M_{0}\right) M_{1} \ldots M_{m} \rightarrow_{\Phi}^{+} N(m \geq 1)$.

Either $M \rightarrow_{\Phi}^{*}\left(\lambda x . N_{0}\right) N_{1} \ldots N_{m} \rightarrow_{\Phi} N_{0}\left[N_{1 / x}\right] N_{1} \ldots N_{m} \rightarrow_{\Phi}^{*} N$ or $M \rightarrow_{\Phi}^{+}$ $\left(\lambda x . N_{0}\right) N_{1} \ldots N_{m} \equiv N$, where $M_{k} \rightarrow{ }_{\Phi}^{+} N_{k}$ or $M_{k} \equiv N_{k}(1 \leq k \leq m)$.

If $M_{1} \rightarrow_{\Phi}^{+} N_{1}$ then $\left\langle N_{1}\right\rangle\left\langle\left\langle M_{1}\right\rangle\right.$ and the proof follows by induction.

Otherwise $M_{1} \equiv N_{1}$ and $M_{0}\left[M_{1} / x\right] M_{2} \ldots M_{m} \rightarrow \stackrel{+}{\Phi} N_{0}\left[M_{1} / x\right] N_{2} \ldots N_{m}$, hence $\left\langle M_{0}\left[M_{1} / x\right] M_{2} \ldots M_{m}\right\rangle<\left\langle N_{0}\left[M_{1} / x\right] N_{2} \ldots N_{m}\right\rangle$ and the proof follows immediately, in all cases.

It is possible to characterize the terms for which the weight is defined.

Corollary 14. $\langle M\rangle$ is defined if and only if $M \rightarrow_{\Phi}^{*} R$, for some $R \in \Upsilon$.

Proof. $\Leftarrow$ The proof follows by Lemma 13. (i) and Lemma 13. (ii).

$\Rightarrow$ The proof is given by induction on $\langle M\rangle$.

If $M \equiv \lambda x \cdot M_{0}$ or $M \equiv x M_{1} \ldots M_{m}(m \in \mathbb{N})$ then the proof follows by induction. Let $M \equiv\left(\lambda z \cdot M_{0}\right) M_{1} \ldots M_{m}(m \geq 1)$, so $\left\langle M_{1}\right\rangle<\langle M\rangle$ and by induction $M_{1} \rightarrow_{\Phi}^{*} S \in \Upsilon$.

- If $M \in \Phi$-NF then $M_{1} \in \Upsilon$, but $M_{1} \notin \Phi=\Upsilon^{0} \cup$ Var. Furthermore $\left\langle M_{0}\left[M_{1} / x\right] M_{2} \ldots M_{m}\right\rangle<1+\left\langle M_{1}\right\rangle+\left\langle M_{0}\left[M_{1} / x\right] M_{2} \ldots M_{m}\right\rangle=\langle M\rangle$ implies that $M_{0}\left[M_{1} / x\right] M_{2} \ldots M_{m} \rightarrow_{\Phi}^{*} R^{\prime}$, for some $R^{\prime} \in \Upsilon$. The proof follows by definition of $\Upsilon$.

- Otherwise $M \rightarrow_{\Phi}^{+} N$, so the proof follows by Lemma 13.(iii) and by induction.

If the weight of a term is defined, then the weight of all its subterms is also defined. The next lemma proves this statement in some particular cases. 
Lemma 15. (i) If $M[N / z] \rightarrow_{\Phi}^{*} R \in \Upsilon$ then $M \rightarrow_{\Phi}^{*} S \in \Upsilon$.

(ii) If $M N \rightarrow_{\Phi}^{*} R \in \Upsilon$ then $M \rightarrow_{\Phi}^{*} S \in \Upsilon$.

Proof. (i) We will prove that $\langle M[N / z]\rangle \in \mathbb{N}$ implies $\langle M\rangle \in \mathbb{N}$ by induction on $h=\langle M[N / z]\rangle$, thus the proof follows by Corollary 14.

Let $M \equiv \lambda x \cdot M_{0} \cdot\left\langle M_{0}[N / z]\right\rangle \in \mathbb{N}$ implies $\left\langle M_{0}\right\rangle<h$ by induction and the proof follows. If $M \equiv x M_{1} \ldots M_{m}(m \geq 1)$ then, in all cases, $\left\langle M_{i}[N / z]\right\rangle<h$ $(1 \leq i \leq m)$ and the proof follows by induction. If $M \equiv\left(\lambda x . M_{0}\right) M_{1} \ldots M_{m}$ $(m \geq 1)$ then $\langle M[N / z]\rangle=1+\left\langle M_{1}[N / z]\right\rangle+\left\langle\left(M_{0}\left[M_{1} / x\right] M_{2} \ldots M_{m}\right)[N / z]\right\rangle$. Thus $\left\langle M_{1}\right\rangle$ and $\left\langle M_{0}\left[M_{1} / x\right] M_{2} \ldots M_{m}\right\rangle$ are defined by induction and the proof follows.

(ii) We will prove that $\langle M N\rangle \in \mathbb{N}$ implies $\langle M\rangle \in \mathbb{N}$ by induction on $\langle M N\rangle$, thus the proof follows by Corollary 14 .

If $M \equiv \lambda x \cdot M_{0}$ then $\langle M N\rangle=1+\langle N\rangle+\left\langle M_{0}[N / z]\right\rangle$, so $\left\langle M_{0}[N / z]\right\rangle \in \mathbb{N}$ and the proof follows by the previous point of this Theorem and the definition of weight. The other cases are easier.

Theorem 16. (i) $M$ is potentially $\Phi$-valuable implies that $M \rightarrow_{\Phi}^{*} R \in \Upsilon$, for some $R \in \Upsilon$.

(ii) $M$ is $\Phi$-solvable implies that $M$ is potentially $\Phi$-valuable.

Proof. (i) $M$ is potentially $\Phi$-valuable means that there is a substitution s, replacing variables by closed terms belonging to $\Phi$, such that $\mathbf{s}(M) \rightarrow_{\Phi}^{*} N \in$ $\Phi$. Since $\Phi \subseteq \Upsilon$, the proof follows by Lemma 15)(i).

(ii) $M$ is $\Phi$-solvable means that there is a $\Phi$-valuable context $C[.] \equiv(\lambda \vec{x} .[].) \vec{N}$ such that $C[M] \rightarrow_{\Phi}^{*} I$. Since $C[M] \rightarrow_{\Phi}^{*} I$ implies $C[M] I \ldots I \rightarrow_{\Phi}^{*} I$, we can assume $\|\vec{x}\| \leq\|\vec{N}\|$ without loss of generality. Moreover $C[M] \rightarrow_{\Phi}^{*} I$ implies $C[M][N / z] \rightarrow_{\Phi}^{*} I \equiv I[N / z]$ for all $N \in \Phi^{0}$, so we can also assume that $C[M] \in \Lambda^{0}$.

If $\vec{N} \equiv \vec{N}_{0} \vec{N}_{1}$ and $\|\vec{x}\|=\left\|\vec{N}_{0}\right\|$ then $C[M] \rightarrow_{\Phi}^{*} M\left[\vec{N}_{0} / \vec{x}\right] \vec{N}_{1} \rightarrow_{\Phi}^{*} I \in \Phi$, thus $M\left[\vec{N}_{0} / \vec{x}\right] \rightarrow_{\Phi}^{*} S^{\prime} \in \Upsilon^{0}$, by Lemma 15.(ii). The proof is done, since $\Upsilon^{0}=\Phi^{0}$.

\subsection{Strong $\Lambda$-Normalization and $\Phi$-Reduction}

In order to prove both that the terms strongly $\Lambda$-normalizing are also $\Phi$-solvable and that terms which $\Phi$-reduce to an element of $\Upsilon$ are strongly $\Lambda$-normalizing, we will use an intersection type assignment system [1, 4] that types all and only the strongly $\Lambda$-normalizing terms $[5,12$.

Definition 17. (i) Let C be a countable set of type-constants (ranging over $\alpha, \beta, .$.$) . The set T(\mathrm{C})$ of types, ranging over by $\sigma, \tau, \pi, \rho, .$. is inductively defined as follows:

$$
\begin{array}{lll}
\sigma \in \mathrm{C} & \Rightarrow & \sigma \in T(\mathrm{C}) \\
\sigma, \tau \in T(\mathrm{C}) & \Rightarrow & (\sigma \rightarrow \tau) \in T(\mathrm{C}) \\
\sigma, \tau \in T(\mathrm{C}) & \Rightarrow & (\sigma \wedge \tau) \in T(\mathrm{C}) .
\end{array}
$$

We use the convention that the constructor $\wedge$ take precedence over $\rightarrow$. 
(ii) $A$ basis is a partial function from Var to $T(\mathrm{C})$ having a finite domain of definition. If $B$ is a basis then $B[\sigma / x]$ denotes the basis such that

$$
B[\sigma / x](y)= \begin{cases}\sigma & \text { if } y \equiv x \\ B(y) & \text { otherwise }\end{cases}
$$

Furthermore, the basis $B$ such that $\operatorname{dom}(B)=\left\{x_{1}, \ldots, x_{n}\right\}$ and $B\left(x_{i}\right)=\sigma_{i}$, for $1 \leq i \leq n$ will be often denoted by $\left[\sigma_{1} / x_{1}, \ldots, \sigma_{n} / x_{n}\right]$.

(iii) The type assignment system $\vdash$ is a formal system proving typing judgments of the shape:

$$
B \vdash M: \sigma
$$

where $M$ is a term, $\sigma \in T(\mathrm{C})$ and $B$ is a basis.

The type assignment system $\vdash$ consists of the following rules:

$$
\begin{array}{cc}
\overline{B[\sigma / x] \vdash x: \sigma}(\text { var }) & \\
\frac{B[\sigma / x] \vdash M: \tau}{B \vdash \lambda x . M: \sigma \rightarrow \tau}(\rightarrow I) & \frac{B \vdash M: \sigma \rightarrow \tau \quad B \vdash N: \sigma}{B \vdash M N: \tau} \\
\frac{B \vdash M: \sigma \quad B \vdash M: \tau}{B \vdash M: \sigma \wedge \tau}(\wedge I) & \\
\frac{B \vdash M: \sigma \wedge \tau}{B \vdash M: \sigma}\left(\wedge E_{l}\right) & \frac{B \vdash M: \sigma \wedge \tau}{B \vdash M: \tau}\left(\wedge E_{r}\right)
\end{array}
$$

If $B, B^{\prime}$ are bases then $B \cap B^{\prime}$ is the basis defined as follows:

$$
\left(B \cap B^{\prime}\right)(y)= \begin{cases}B(y) \wedge B^{\prime}(y) & \text { if both } B(y) \text { and } B^{\prime}(y) \text { are defined, } \\ B(y) & \text { if } B(y) \text { is defined and } B^{\prime}(y) \text { is undefined, } \\ B^{\prime}(y) & \text { if } B^{\prime}(y) \text { is defined and } B(y) \text { is undefined, } \\ \text { undefined } & \text { otherwise. }\end{cases}
$$

The type systems $\vdash$ enjoys the subject-reduction property and a restricted form of subject-expansion.

Property 18 (Subject-Reduction and Typed Subject-Expansion).

(i) If $B \vdash M: \sigma$ and $M \rightarrow_{\Lambda} N$ then $B \vdash N: \sigma$.

(ii) Let $C\left[\right.$.] be a context. Then $B \vdash C[P[Q / x]]: \sigma$ and $B^{\prime} \vdash Q: \tau$ imply $B \cap B^{\prime} \vdash C[(\lambda x . P) Q]: \sigma$.

Proof. See [4. 
Lemma 19. If $M \in \Upsilon$ then $B \vdash M: \sigma$, for some basis $B$ and $\sigma \in T(\mathrm{C})$.

Proof. The proof is given by induction on the stratification of $\Upsilon$. The case $M \in$ $\Upsilon_{0}$ is trivial. If $M \in \Upsilon_{i+1}$, the cases $M \equiv x M_{1} \ldots M_{m}$ and $M \equiv \lambda x . P$ follow easily from the inductive hypothesis, using the rules of the system. If $M \equiv$ $(\lambda x . P) Q M_{0} \ldots M_{m}$ then both $Q \in \Upsilon_{i}-\left(\Lambda^{0} \cup\right.$ Var $)$ and $P[Q / x] M_{1} \ldots M_{m} \rightarrow_{\Phi_{i}}^{*}$ $R \in \Upsilon_{i}$. Therefore $B \vdash R: \sigma$, by induction. Since the $\rightarrow_{\Phi_{i}}^{*}$ reduction reduces only in case the argument belongs to $\Phi_{i}$ this can be typed by induction, and hence $B \vdash P[Q / x] M_{1} \ldots M_{m}: \sigma$ by Property [18.(ii). Since $B^{\prime} \vdash Q: \tau$ by induction, the proof follows by Property 18 (ii) .

Theorem 20. $M \rightarrow_{\Phi}^{*} R \in \Upsilon$ implies $M$ is $\Lambda$-strongly normalizing.

Proof. By Lemma [19, $B \vdash R: \sigma$ for some basis $B$ and $\sigma \in T(\mathrm{C})$. Thus $B \vdash$ $M: \sigma$ by Property [18,(ii). Then the proof follows from the fact that the system characterizes the strongly $\Lambda$-normalizing terms [5, 12].

\subsection{Strong $\Lambda$-Normalization and $\Phi$-Solvability}

In order to prove that $\Lambda$-strong normalization implies $\Phi$-solvability, we will use a computability argument, which is an adaptation to intersection types of the reducibility candidates method [3].

Let $M$ be a term, let $\mathrm{FV}(M) \subseteq\left\{x_{1}, \ldots, x_{m}\right\}$ for some $m \geq 0$ and let $O^{i}$ be $\lambda x_{0} \ldots x_{i} . x_{i}$ for all $i \in \mathbb{N}$.

The meaning of $\mathcal{P}(M)$ will be: "there is $r \in \mathbb{N}$ such that, for all $h, k \geq r$,

$$
M\left[O^{h} / x_{1}, \ldots, O^{h} / x_{m}\right] \underbrace{O^{h} \ldots O^{h}}_{k} \rightarrow_{\Phi}^{*} O^{s} \text { for some } s \in \mathbb{N} . "
$$

Property 21. (i) $\mathcal{P}(M)$ implies $M$ is $\Phi$-solvable.

(ii) $\mathcal{P}(x \vec{M})$ and $\mathcal{P}(N)$ imply $\mathcal{P}(x \vec{M} N)$.

Proof. (i) $M\left[O^{h} / x_{1}, \ldots, O^{h} / x_{m}\right] \underbrace{O^{h} \ldots O^{h}}_{k} \rightarrow_{\Phi}^{*} O^{s}$ implies that

$$
M\left[O^{h} / x_{1}, \ldots, O^{h} / x_{m}\right] \underbrace{O^{h} \ldots O^{h}}_{k} \underbrace{I \ldots \ldots I}_{s} \rightarrow_{\Phi}^{*} I .
$$

(ii) Let $\vec{M} \equiv M_{1} \ldots M_{n}(n \in \mathbb{N})$ and let $\mathrm{FV}(x \vec{M} N) \subseteq\left\{x_{1}, \ldots, x_{m}\right\}(m \in \mathbb{N})$.

$$
\begin{aligned}
& \exists r_{0} \in \mathbb{N}, \forall h_{0}, k_{0} \geq r_{0}, \exists s_{0} \in \mathbb{N}, x \vec{M}\left[O^{h_{0}} / x_{1}, \ldots, O^{h_{0}} / x_{m}\right] \underbrace{O^{h_{0}} \ldots O^{h_{0}}}_{k_{0}} \rightarrow_{\Phi}^{*} O^{s_{0}}, \\
& \exists r_{1} \in \mathbb{N}, \forall h_{1}, k_{1} \geq r_{1}, \exists s_{1} \in \mathbb{N}, N\left[O^{h_{1}} / x_{1}, \ldots, O^{h_{1}} / x_{m}\right] \underbrace{O^{h_{1}} \ldots O^{h_{1}}}_{k_{1}} \rightarrow_{\Phi}^{*} O^{s_{1}},
\end{aligned}
$$

by hypothesis. So the proof follows by putting $r=\max \left\{r_{0}, r_{1}, n+1\right\}$.

The predicate $\mathcal{P}$ is used to define the computability predicate. 
Definition 22. The predicate Comp is defined by induction on types as follows:

- $\operatorname{Comp}(B, \alpha, M)$ if and only if $\mathcal{P}(M), \alpha \in C$ and $B$ is a basis;

$-\operatorname{Comp}(B, \sigma \rightarrow \tau, M)$ if and only if, for all $N \in \Lambda, C \operatorname{Comp}\left(B^{\prime}, \sigma, N\right)$ implies $\operatorname{Comp}\left(B \cap B^{\prime}, \tau, M N\right)$;

- $\operatorname{Comp}(B, \sigma \wedge \tau, M)$ if and only if $\operatorname{Comp}(B, \sigma, M)$ and $\operatorname{Comp}(B, \tau, M)$.

We prove that $B \vdash M: \sigma$ implies $\operatorname{Comp}(B, \sigma, M)$, which in its turn implies $\mathcal{P}(M)$. It is easy to check that $\operatorname{Comp}(B, \sigma, M)$ does not imply $B \vdash M: \sigma$.

Lemma 23. (i) $\mathcal{P}(x \vec{M})$ implies $\operatorname{Comp}(B, \sigma, x \vec{M})$, for all $B$ and $\sigma \in T(\mathrm{C})$.

(ii) $\operatorname{Comp}(B, \sigma, M)$ implies $\mathcal{P}(M)$, for all $B$ and $\sigma \in T(\mathrm{C})$.

Proof. The proof is by mutual induction on $\sigma$. The only case which is not obvious is when $\sigma=\tau \rightarrow \rho$.

(i) We will prove that $\operatorname{Comp}\left(B^{\prime}, \tau, N\right)$ implies $\operatorname{Comp}\left(B \cap B^{\prime}, \rho, x \vec{M} N\right)$, thus $\operatorname{Comp}(B, \tau \rightarrow \rho, x \vec{M})$ follows by definition. $\operatorname{Comp}\left(B^{\prime}, \tau, N\right)$ implies $\mathcal{P}(N)$, by induction. But $\mathcal{P}(x \vec{M})$ by hypothesis, thus $\mathcal{P}(x \vec{M} N)$ by Property 21.(ii).

(ii) $\mathcal{P}(z)$ holds so, in particular, $\operatorname{Comp}\left(B^{\prime}, \tau, z\right)$ holds by induction on (i). Thus $\operatorname{Comp}\left(B \cap B^{\prime}, \rho, M z\right)$ by definition of Comp and this implies $\mathcal{P}(M z)$ by induction. That is, there is $r \in \mathbb{N}$ such that, for all $h, k \geq r$,

$$
M z\left[O^{h} / z, O^{h} / x_{1}, \ldots, O^{h} / x_{m}\right] \underbrace{O^{h} \ldots O^{h}}_{k} \rightarrow_{\Phi}^{*} O^{s}
$$

where $s \in \mathbb{N}, O^{h} \equiv \lambda x_{0} \ldots x_{h} \cdot x_{h}$ and $\mathrm{FV}(M z) \subseteq\left\{z, x_{1}, \ldots, x_{m}\right\}(m \geq 0)$. So, if $r^{\prime}=r+1$, for all $h, k \geq r^{\prime}, M\left[O^{h} / x_{1}, \ldots, \bar{O}^{h} / x_{m}\right] \underbrace{O^{h} \ldots . O^{h}}_{k} \rightarrow_{\Phi}^{*} O^{s}$, for some $s$. Hence $\mathcal{P}(M)$ holds.

Property 24. Let $Q$ be such that $\mathcal{P}(Q)$.

If $\operatorname{Comp}(B, \sigma, P[Q / x] \vec{Q})$ then $\operatorname{Comp}(B, \sigma,(\lambda x . P) Q \vec{Q})$.

Proof. The proof is by induction on the structure of types.

Assume $\sigma \in \mathrm{C}$. Note that $\mathcal{P}(Q)$ and Lemma 15.(i) imply

$$
\exists r_{0} \in \mathbb{N}, \forall h_{0}, k_{0} \geq r_{0}, \quad Q\left[O^{h_{0}} / x_{1}, \ldots, O^{h_{0}} / x_{m}\right] \rightarrow_{\Phi}^{*} M \in \Upsilon^{0}=\Phi^{0} .
$$

Moreover, $\operatorname{Comp}(B, \sigma, P[Q / x] \vec{Q})$ implies $\mathcal{P}(P[Q / x] \vec{Q})$ by definition, thus

$$
\exists r_{1}, \forall h_{1}, k_{1} \geq r_{1}, \exists s_{1},(P[Q / x] \vec{Q})\left[O^{h_{1}} / x_{1}, \ldots, O^{h_{1}} / x_{m}\right] \underbrace{O^{h_{1}} \ldots \ldots O^{h_{1}}}_{k_{1}} \rightarrow{ }_{\Phi}^{*} O^{s_{1}} .
$$

Let $r=\max \left\{r_{0}, r_{1}\right\}$. Then, $\forall h, k \geq r,((\lambda x . P) Q \vec{Q})\left[O^{h} / x_{1}, \ldots, O^{h} / x_{m}\right] \underbrace{O^{h} \ldots . O^{h}}_{k}$ $=_{\Phi}(P[Q / x] \vec{Q})\left[O^{h} / x_{1}, \ldots, O^{h} / x_{m}\right] \underbrace{O^{h} \ldots O^{h}}_{k} \rightarrow_{\Phi}^{*} O^{s}$, for some $s \in \mathbb{N}$.

Hence $\mathcal{P}(Q)$ and $\sigma \in \mathrm{C}$ imply $\operatorname{Comp}(B, \sigma,(\lambda x . P) Q \vec{Q})$. 
Let $\sigma=\tau \rightarrow \rho$. Thus $\operatorname{Comp}(B, \tau \rightarrow \rho, P[Q / x] \vec{Q})$ implies that $\forall N$ such that $\operatorname{Comp}\left(B^{\prime}, \tau, N\right), \operatorname{Comp}\left(B \cap B^{\prime}, \rho, P[Q / x] \vec{Q} N\right)$ holds. Therefore by induction, $\operatorname{Comp}\left(B \cap B^{\prime}, \rho,(\lambda x . P) Q \vec{Q} N\right)$ and hence $\operatorname{Comp}\left(B \cap B^{\prime}, \tau \rightarrow \rho,(\lambda x . P) Q \vec{Q}\right)$ by definition of Comp. The case $\sigma=\tau \wedge \rho$ is trivial.

Lemma 25. Let $\mathrm{FV}(M) \subseteq\left\{x_{1}, \ldots, x_{n}\right\}$ and $B\left(x_{i}\right)=\sigma_{i}(1 \leq i \leq n)$. If $\operatorname{Comp}\left(B_{i}, \sigma_{i}, N_{i}\right)(1 \leq i \leq n)$ and $B \vdash M: \tau$, then

$$
\operatorname{Comp}\left(B_{1} \cap \ldots \cap B_{n}, \tau, M\left[N_{1} / x_{1}, \ldots, N_{n} / x_{n}\right]\right) .
$$

Proof. By induction on the derivation $d$ of $B \vdash M: \tau$. The most interesting case is when the last rule applied on $d$ is $(\rightarrow I)$. Let $M \equiv \lambda x \cdot M^{\prime}, \tau=\mu \rightarrow \rho$ and

$$
\frac{B[\mu / x] \vdash M^{\prime}: \rho}{B \vdash \lambda x \cdot M^{\prime}: \mu \rightarrow \rho}(\rightarrow I)
$$

Suppose that $\operatorname{Comp}\left(B^{\prime}, \mu, N\right)$ holds. Then $\mathcal{P}(N)$ holds by Lemma 23. (ii). Thus, by induction

$$
\operatorname{Comp}\left(B^{\prime} \cap B_{1} \cap \ldots \cap B_{n}, \rho, M^{\prime}\left[N_{1} / x_{1}, \ldots, N_{n} / x_{n}, N / x\right]\right)
$$

and $\operatorname{Comp}\left(B^{\prime} \cap B_{1} \cap \ldots \cap B_{n}, \rho,\left(\lambda x \cdot M^{\prime}\left[N_{1} / x_{1}, \ldots, N_{n} / x_{n}\right]\right) N\right)$ by Property 24 . Hence, $\operatorname{Comp}\left(B_{1} \cap \ldots \cap B_{n}, \mu \rightarrow \rho, M\left[N_{1} / x_{1}, \ldots, N_{n} / x_{n}\right]\right)$ by definition of Comp. All other cases follow directly from the inductive hypothesis.

Theorem 26. $M$ is $\Lambda$-strongly normalizing implies that $M$ is $\Phi$-solvable.

Proof. In [12,5] it is proved that the system $\vdash$ characterizes the strongly $\Lambda$ normalizing terms. So, let $B \vdash M: \sigma, \mathrm{FV}(M) \subseteq\left\{x_{1}, \ldots, x_{n}\right\}$ and $B\left(x_{i}\right)=\sigma_{i}$. Since $\operatorname{Comp}\left(B, \sigma_{i}, x_{i}\right)(1 \leq i \leq n)$ by Lemma 23. (i), then $\operatorname{Comp}(B, \sigma, M)$ by Lemma 25. Thus $\mathcal{P}(M)$ by Lemma 23. (ii). The proof follows by Property 21. (i).

\section{References}

1. van Bakel S., Intersection type assignment systems, Theoretical Computer Science, 38(2):246-269, Elsevier, 1997.

2. Barendregt H.P., The Lambda Calculus: its syntax and semantics, N.103 in Studies in Logic and the Foundations of Mathematics (revised edition), North-Holland, Amsterdam, 1994.

3. Coppo M., Dezani-Ciancaglini M., Zacchi M., Type Theories, Normal Forms and $\mathcal{D}_{\infty}$ Lambda Models, Information and Control, 72, 2, 1987, pp.85-116.

4. Coppo M., Dezani-Ciancaglini M., An Extension of the Basic Functionality Theory for the $\lambda$-Calculus Notre-Dame Journal of Formal Logic, 21(4), pp. 685-693, October 1980.

5. Krivine J.L., Lambda-Calculus, Types and Models, Ellis Horwood Series in Computers and Their Applications. 1993. 
6. Landin P.J., The mechanical evaluation of expressions, Computer Journal, 1964.

7. Paolini L., Call-by-value separability and computability, ICTCS'01, Restivo, Ronchi Della Rocca, and Roversi, eds, LNCS 2202, Springer-Verlag, 74-89.

8. Paolini L., Pimentel E., Ronchi Della Rocca S. Lazy strong normalization, ITRS'04, ENTCS vol. 136, pp. 103-116, 2005.

9. Paolini L., Ronchi Della Rocca S., Call-by-value Solvability, Theoretical Informatics and Applications, 33(6), 507-534, 1999.

10. Paolini L., Ronchi Della Rocca S., The Parametric Parameter Passing $\lambda$-calculus, Information and Computation, 189(1):87-106, 2004.

11. Plotkin G.D., Call-by-name, call-by-value and the $\lambda$-calculus, Theoretical Computer Science (1) 125-159, 1975.

12. Pottinger G., A type assignment for the strongly normalizable $\lambda$-terms, in To H.B. Curry: essays on combinatory logic, lambda calculus and formalism, pp.561-577, Academic Press, London, 1980.

13. Ronchi Della Rocca S., Paolini L., The Parametric $\lambda$-calculus. A meta-model for computation, Texts in Theoretical Computer Science: an EATCS Series, SpringerVerlag, Berlin, 2004. 